\title{
Giant cardiac myxoma: Real-time characterization by 64-slice computed tomography
}

\author{
Luigi Muzzi, MD, ${ }^{\mathrm{a}, \mathrm{c}, \mathrm{d}}$ Giuseppe Pugliese, MD, ${ }^{\mathrm{a}}$ Ilaria D'Angeli, MD, ${ }^{\mathrm{a}}$ Riccardo Ferrari, MD, ${ }^{\mathrm{b}}$ \\ Andrea Laghi, MD, ${ }^{\mathrm{b}} \mathrm{D}$. Rose, MD, ${ }^{\mathrm{a}} \mathrm{F}$. Vecchietti, MD, ${ }^{\mathrm{b}}$ and Giacomo Frati, MD, ${ }^{\mathrm{a}, \mathrm{c}, \mathrm{d}}$ \\ Rome and Pozzilli, Italy
}

Video clip is available online.

performed to confirm the diagnosis. Multiplanar reconstructions exactly defined the tumor attachment in the angle between the upper portion of interatrial septum and the left atrial roof (Figure 1). Functional and dynamic features were obtained by 3-dimensionally rendered real-time recon-

\section{CLINICAL SUMMARY}

A 70-year-old woman was admitted at our institution because of recent onset of palpitations, dyspnea, and fatigue. On physical examination, she appeared well and she did not present particular signs except for a pulse rate of 114 beats per minute; her blood pressure was $155 / 85 \mathrm{~mm} \mathrm{Hg}$, axillary temperature was $36.7^{\circ} \mathrm{C}$, respirations were 16 breaths per minute, and arterial blood gas measurements were normal. The chemistry and hematologic laboratory values were within the normal reference ranges. Electrocardiography revealed sinus tachycardia at a rate of 115 beats per minute and diphasic $\mathrm{P}$ waves in leads II, aVF, and $\mathrm{V}_{3}$ through $\mathrm{V}_{5}$. A chest radiography showed no abnormalities of the heart and mediastinum, and the lungs were clear. However, in the lateral view, an egg-shaped image corresponding to the atria was present and appeared highly suspicious for an intracardiac mass.

A transthoracic echocardiographic examination disclosed a dilated left atrium $(60 \times 40 \mathrm{~mm})$ filled almost entirely by a mobile, pedunculated mass of echoes, 2.7 by $7 \mathrm{~cm}$, that partially prolapsed into the left ventricle during diastole with an incomplete closure of the mitral valve leaflets and an associated jet of mild regurgitation (Figure 1). The mass was highly mobile and appeared to be attached to the interatrial septum by a large stalk $(2 \mathrm{~cm})$. Transesophageal echocardiogram was impracticable because of the presence of Zenker's diverticulum.

A 64-slice contrast-enhanced computed tomography (LightSpeed VCT, GE Healthcare, Milwaukee, Wis) was

\footnotetext{
From the Cardiac Surgery Unit ${ }^{\mathrm{a}}$ and the Department of Radiology, ${ }^{\mathrm{b}}$ University of Rome "Sapienza," Polo Pontino, ICOT Institute, Rome, Italy; the Spencer Lorillard Foundation, Rome, Italy ${ }^{\mathrm{c}}$; and IRCCS Neuromed, ${ }^{\mathrm{d}}$ Pozzilli, Italy.

Received for publication March 6, 2008; accepted for publication March 21, 2008; available ahead of print Aug 26, 2008.

Address for reprints: Luigi Muzzi, MD, Department of Heart and Great Vessels, Cardiac Surgery Unit Polo Pontino, ICOT Institute, Via F. Faggiana, 34, 04100 Latina, Italy (E-mail: luigimuzzi@hotmail.com).

J Thorac Cardiovasc Surg 2009;138:493-5

$0022-5223 / \$ 36.00$

Copyright (c) 2009 by The American Association for Thoracic Surgery

doi:10.1016/j.jtcvs.2008.03.063
} demonstrated this huge mass prolapsing through the mitral orifice and greatly reducing the diastolic filling of the ventricle (Video 1).

The patient had an urgent operation. Because of the large dimensions and the site of implant, we preferred a biatrial approach; thus the resection was accomplished in a "septal superior approach fashion.' Actually, by direct visualization, the mass presented a large stalk localized in the upper interatrial septum and widely extending to the roof of the left atrium. The tumor was extracted en bloc, and gross inspection showed a huge trilobulated mass measuring about $4 \times 7 \times 3 \mathrm{~cm}$ with a stalk of $2 \times 2 \mathrm{~cm}$ (Figure 2). Fresh section revealed a reddish mass with diffuse hemorrhagic infiltration strongly suggestive of cardiac myxoma. Once the mass had been removed, the mitral apparatus was carefully inspected for possible valve incompetence that was preoperatively undetectable. However, the saline solution injection revealed only a trivial central regurgitation not susceptible of surgical correction.

The procedure was completed as usual without complications, and the postoperative course was uneventful except for the occurrence of a junctional rhythm, which spontaneously resolved in the third postoperative day. Histology confirmed the diagnosis of myxoma.

\section{DISCUSSION}

Usually pedunculated, cardiac myxomas develop in the atria in about $90 \%$ of the cases $(75 \%$ left atrium and $15 \%$ to $20 \%$ right atrium). Left atrial myxomas originate from the interatrial septum in $85 \%$ of the cases (mostly from the fossa ovalis), with the others usually arising from the posterior or anterior atrial wall. ${ }^{1}$

Giant cardiac myxomas have also been reported in the literature, and when sited in the left atrium, they can give rise to critical drawbacks for diagnosis of coexistent or subsequent mitral valve disease. ${ }^{2}$ Multislice computed tomography is emerging as a useful adjunctive tool in cardiovascular 

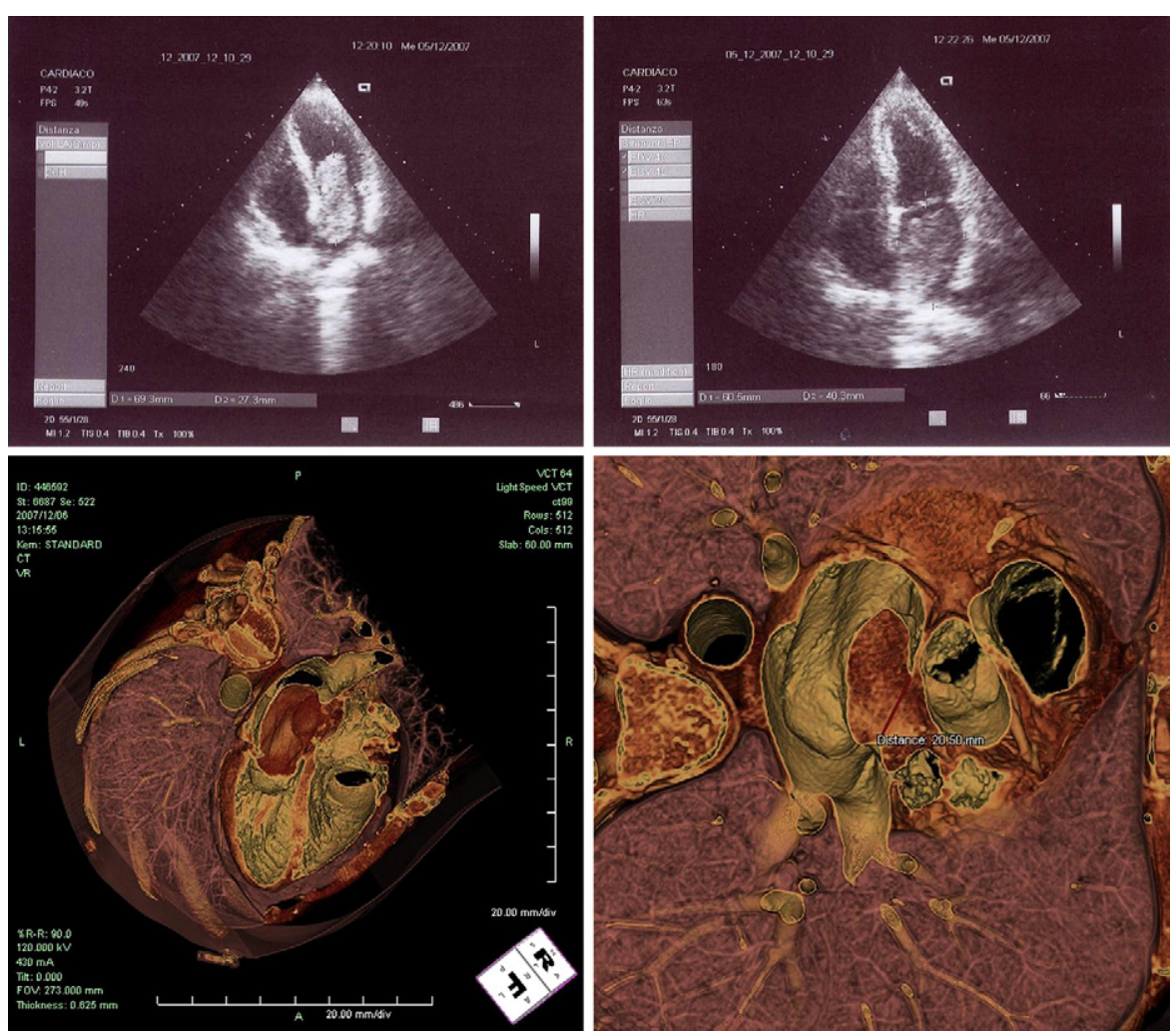

FIGURE 1. Preoperative transthoracic echocardiography showed a huge left atrial mass prolapsing into the left ventricle. Sixty-four-slice computed tomography. Three-dimensional, volume-rendered reconstruction demonstrating the left atrial mass and the diastolic obstruction of the mitral inflow (left-hand panel). Cross-sectional reconstruction at the level of upper interatrial septum revealed the tumor attachment (right-hand panel).

imaging, capable of supplying an extremely accurate, operator-independent definition of anatomic and functional details. ${ }^{3}$ Its great diagnostic power can sometimes cause this technique to be used inappropriately. In our experience, pre- cisely identifying the unusual tumor attachment resulted in a fundamental diagnostic implement for the choice of surgical approach, which is crucial to achieve a radical excision, to avoid the risk of tumor fragmentation (especially for huge
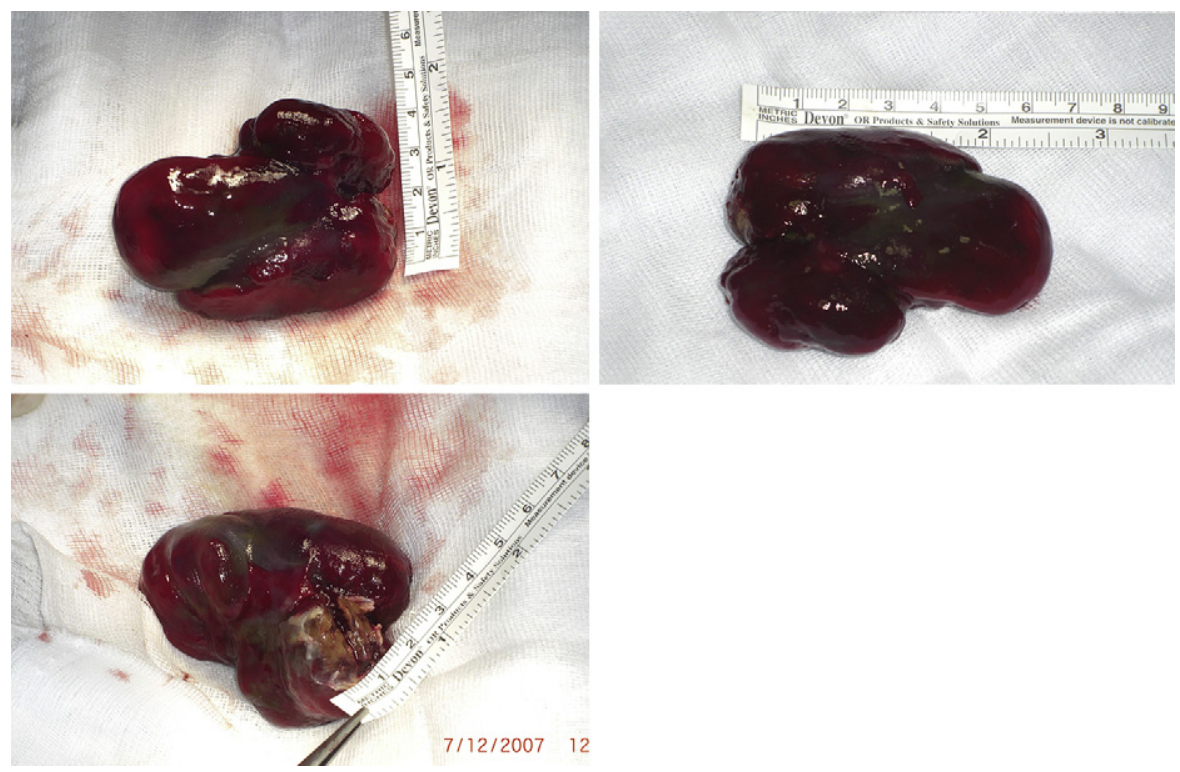

FIGURE 2. Intraoperative pictures of the myxoma after its en bloc resection. 
myxomas), and to manage a possibly associated valve disease. $^{2}$

\section{References}

1. Reyen K. Cardiac myxomas. N Engl J Med. 1995;333:1610-7.
2. Davoli G, Muzzi L, Lucchese G, Uricchio N, Chiavarelli M. Large left atrial myxoma with severe mitral valve regurgitation. The inverted $\mathrm{T}$-shaped biatrial incision revisited. Tex Heart Inst J. 2006;33:51-3.

3. Feuchtner G, Mueller S, Bonatti J, Friedrich G, zur Nedden D, Smekal A. Prolapsing atrial myxoma. Dynamic visualization with multislice computed tomography. Circulation. 2004;109:e165-6.

\title{
An unusual case of a strangulated lung hernia after an Ivor Lewis esophagectomy
}

\author{
Rony Atoui, MD, MSc, Lawrence Lee, and Lorenzo Ferri, MD, PhD, Montreal, Quebec, Canada
}

Intercostal lung herniation is a rare condition that most commonly occurs after a traumatic event or through a congenital defect. We report an unusual case of a right lung hernia after an Ivor Lewis esophagectomy that was found to be strangulated and necrotic, requiring a wedge resection.

\section{CLINICAL SUMMARY}

A 30-year-old morbidly obese man with distal esophageal adenocarcinoma underwent an uneventful Ivor Lewis esophagectomy and thereafter had 3 cycles of docetaxel (Taxotere), cisplatin, and 5-flourouracil chemotherapy. An en-bloc resection with 2-field lymphadenectomy was performed through a laparotomy and right thoracotomy, and a hand-sewn esophagogastric anastomosis was generated cephalad to the azygous vein. The ribs were reapproximated with 4 interrupted figure eight, no. 1, nonlooped polydioxanone sutures. Postoperatively, the patient actively and enthusiastically participated in chest physiotherapy, including early ambulation and forced coughing. On postoperative day 8 , he had intermittent low-grade fever; however, a barium swallow and a chest computed tomographic (CT) scan did not reveal an anastomotic leak or mediastinal abscess. On postoperative day 11 , he experienced a sudden onset of right chest pain and dyspnea after an episode of vigorous coughing. Evaluation of the pleuritic pain revealed a localized tender bulge measuring $10 \times 5 \mathrm{~cm}$ at the level of the thoracotomy incision associated with massive subcu-

\footnotetext{
From the Division of Thoracic Surgery, Department of Surgery, McGill University Health Center, Montreal, Quebec, Canada.

Received for publication April 6, 2008; accepted for publication April 13, 2008; available ahead of print Aug 26, 2008.

Address for reprints: Lorenzo Ferri, MD, PhD, Division of Thoracic Surgery, McGill University Health Center, 1650 Cedar Ave, Montreal, Quebec, H3G 1A4, Canada (E-mail: lorenzo.ferri@muhc.mcgill.ca).

J Thorac Cardiovasc Surg 2009;138:495-6

$0022-5223 / \$ 36.00$

Copyright (c) 2009 by The American Association for Thoracic Surgery doi:10.1016/j.jtcvs.2008.04.026
}

taneous emphysema involving the right chest and neck. A CT scan of the chest revealed a large right pneumothorax associated with a herniation of a segment of the right lung parenchyma (Figure 1). On review of the previous CT scan, an uncomplicated minor lung hernia was evident.

The patient was brought to the operating theater after inserting a thoracostomy tube to relieve the pneumothorax. On entering the thoracic cavity, we confirmed the presence of a strangulated and necrotic right middle lobe hernia entrapped between the surrounding ribs (Figure 2). A wedge resection of this segment was performed, and the ribs were reapproximated with no. 1 looped polydioxanone sutures.

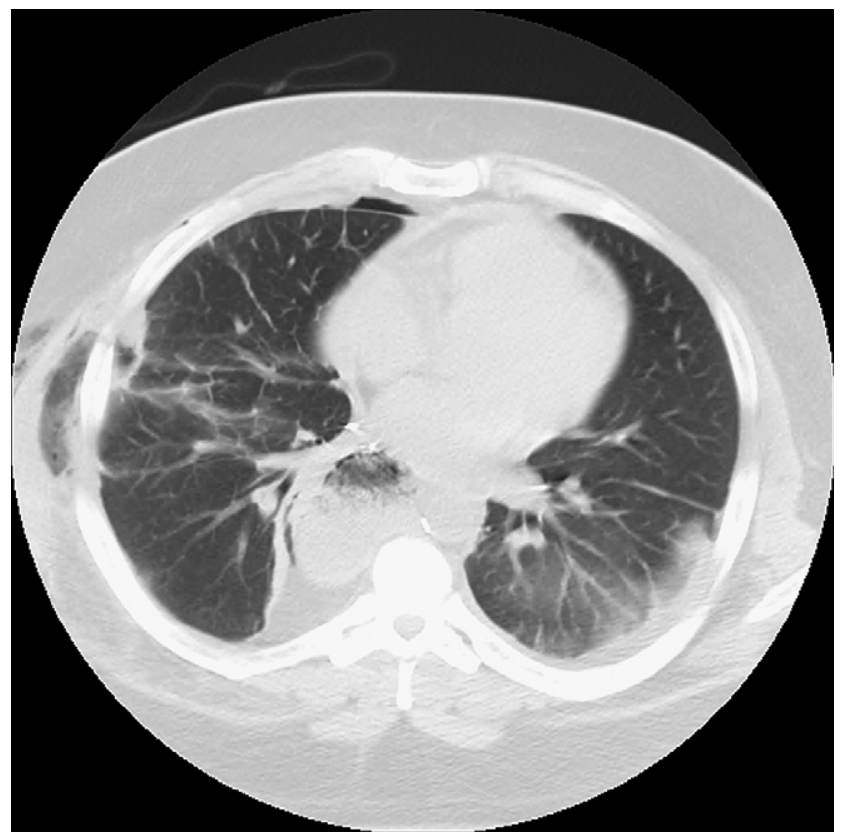

FIGURE 1. Preoperative computed tomographic scan of the chest demonstrating the herniation of a segment of the right lung associated with a pneumothorax. 